\title{
Impact of Time-of-Flight PET/CT with a Large Axial Field of View for Reducing Whole-Body Acquisition Time
}

\author{
Go Akamatsu $^{1,2}$, Koji Uba ${ }^{3}$, Takafumi Taniguchi ${ }^{1}$, Katsuhiko Mitsumoto ${ }^{1}$, Akihiro Narisue ${ }^{3}$, Yuji Tsutsui ${ }^{4}$, \\ and Masayuki Sasaki ${ }^{1}$ \\ ${ }^{1}$ Department of Health Sciences, Graduate School of Medical Sciences, Kyushu University, Fukuoka, Japan; ${ }^{2}$ Division of Molecular \\ Imaging, Institute of Biomedical Research and Innovation, Kobe Japan; ${ }^{3}$ Department of Radiological Technology, Saga University \\ Hospital, Saga, Japan; and ${ }^{4}$ Department of Medical Technology, Kyushu University Hospital, Fukuoka, Japan
}

The aim of this study was to evaluate the imaging performance of 39- and 52-ring time-of-flight (TOF) PET/CT scanners. We also assessed the potential of reducing the scanning time using a 52-ring TOF PET/CT scanner. Methods: PET/CT scanners with 39- and 52-ring lutetium oxyorthosilicate detectors were evaluated. The axial fields of view were 16.2 and $21.6 \mathrm{~cm}$, respectively. We used a National Electrical Manufacturers Association International Electrotechnical Commission body phantom filled with an ${ }^{18} \mathrm{~F}$ solution containing background activity of 5.31 and $2.65 \mathrm{kBq} / \mathrm{mL}$ for the studies. The sphere-to-background ratio was $4: 1$. The PET data were acquired for $10 \mathrm{~min}$ in 3-dimensional list mode and then reconstructed with both ordered-subsets reconstruction maximization and ordered-subsets reconstruction maximization plus point-spread function plus time-of-flight algorithms. PET images with different acquisition times were reconstructed (from 1 to $10 \mathrm{~min}$ ). The image quality was physically assessed using the sensitivity, noise-equivalent counting rate, coefficient of variation of background activity, and relative recovery coefficient. Results: The total system sensitivities of the 39- and 52-ring scanners were 5.6 and $9.3 \mathrm{kcps} / \mathrm{MBq}$, respectively. Compared with the 39-ring scanner, the noise-equivalent counting rate of the 52-ring scanner was $60 \%$ higher for both the high-activity and the low-activity models. The recovery coefficient was consistent, irrespective of the number of detector rings. The coefficient of variation of the 52-ring scanner using a 3-min acquisition time was equivalent to that of the 39-ring scanner using a 4-min acquisition time. Conclusion: The image quality of the 52-ring scanner is superior to that of the 39-ring scanner. The acquisition time per bed position of the 52-ring system can be reduced by about $25 \%$ without compromising image quality. In addition, the number of bed positions required is $25 \%$ lower for the 52 -ring system. Finally, the examination time required for a whole-body PET scan is considered to be reduced by about $40 \%$ if the 52 -ring scanner is used.

Key Words: axial field of view; time-of-flight; PET/CT; image quality; sensitivity

J Nucl Med Technol 2014; 42:101-104

DOI: 10.2967/jnmt.114.140665

Received Mar. 23, 2014; revision accepted Apr. 17, 2014.

For correspondence or reprints contact: Masayuki Sasaki, Division of Medical Quantum Science, Department of Health Sciences, Graduate School of Medical Sciences, Kyushu University, 3-1-1 Maidashi, Higashi-ku, Fukuoka 812-8582, Japan.

E-mail: msasaki@hs.med.kyushu-u.ac.jp

Published online May 5, 2014.

COPYRIGHT (c) 2014 by the Society of Nuclear Medicine and Molecular Imaging, Inc.
$\mathbf{P}$ abolic information useful for patient care and for monitoring the pharmacokinetics of drugs $(1,2)$. High image quality is required for an accurate diagnosis (3). PET image quality is strongly dependent on scanner sensitivity (4), which in turn is associated with the length of the axial field of view (FOV) (4-6). A PET system with a longer axial FOV has a higher sensitivity because there are a large number of detectors (Fig. 1). In addition, the number of bed positions can be reduced because of the large axial range for whole-body PET. PET scanners with a longer axial range can be used to reduce the patient dose, decrease the scanning time, or reduce the image noise while maintaining other constant image characteristics. To date, however, there has been no comparison of actual PET images acquired on systems having different axial FOVs.

In this study, we evaluated the PET image quality of 39- and 52-ring time-of-flight (TOF) PET/CT scanners and assessed the possibility of reducing the whole-body scanning time using a 52-ring TOF PET/CT scanner.

\section{MATERIALS AND METHODS}

\section{PET/CT Scanners with 39- and 52-Ring Detector System}

Two types of Biograph mCT scanner (Siemens Healthcare), one with a 39-ring detector system and the other with a 52-ring detector system, were used in this study (7). The specifications of the 2 PET scanners are shown in Table 1. The PET components of both the 39-ring and the 52-ring scanners are lutetium oxyorthosilicate scintillators $4 \times 4 \times 20 \mathrm{~mm}$ in size. The 39-ring scanner comprises a total of 24,336 lutetium oxyorthosilicate detector elements, covering an axial FOV of $16.2 \mathrm{~cm}$. The 52-ring scanner comprises a total of 32,448 detector elements, covering an axial FOV of $21.6 \mathrm{~cm}$. Both PET scanners operate with a 4.1-ns coincidence time window and a $435-$ to $650-\mathrm{keV}$ energy window.

The CT component of the 39-ring scanner consists of a 128slice multidetector helical CT scanner, whereas that of the 52-ring scanner consists of a 40-slice multidetector helical CT scanner. The CT scan parameters were $120 \mathrm{kV}$, automatic exposure control depending on body habitus, and 0.5 -s rotation of the gantry in both PET/CT scanners. CT was performed to obtain a map for attenuation correction. 


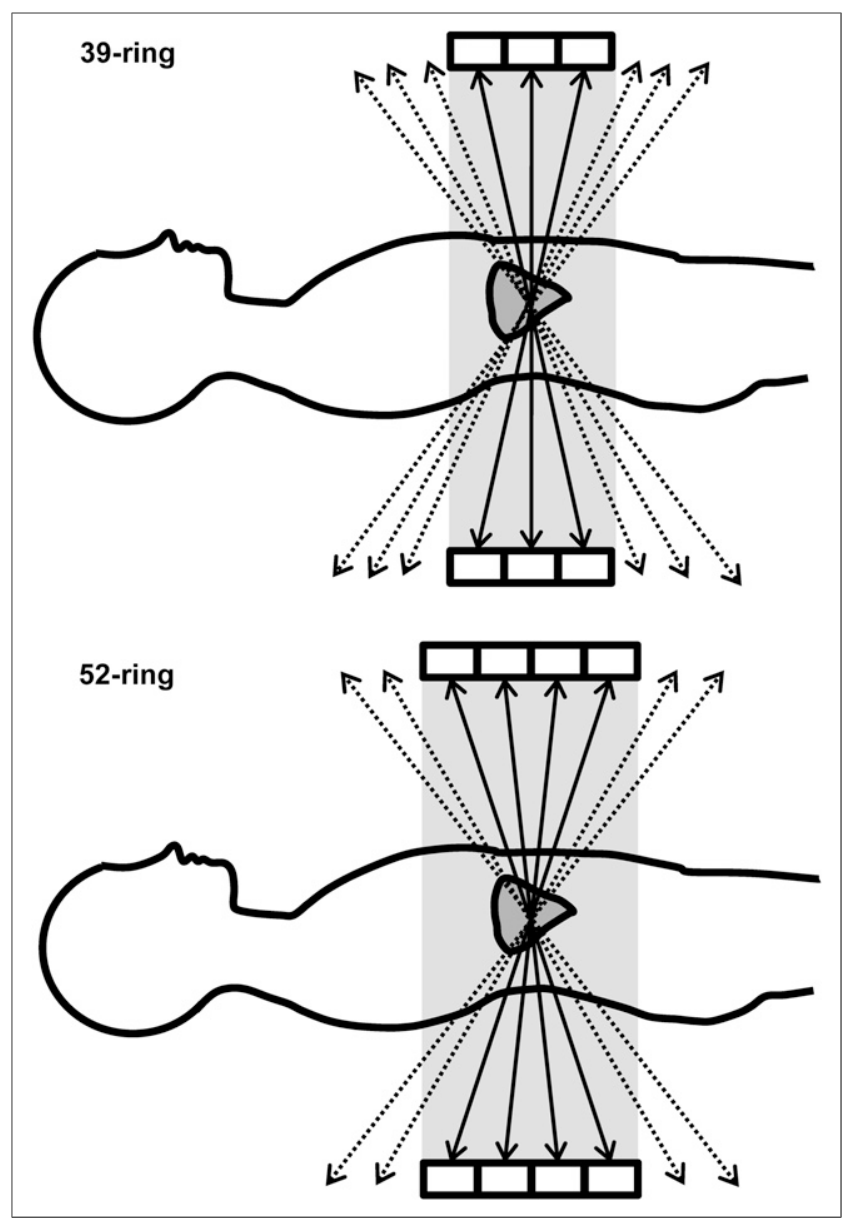

FIGURE 1. A 3D emission acquisition of 39- (upper) and 52-ring (lower) scanners. The 52-ring scanner has large acceptance angle. Thus, system sensitivity of 52-ring scanner is higher than that of 39-ring scanner.

\section{Evaluation of System Sensitivity}

Following the National Electrical Manufacturers Association (NEMA) NU-2 2007 protocol, a phantom with 5 concentric aluminum sleeves $70 \mathrm{~cm}$ in length was used to evaluate the system sensitivity (NEMA PET Sensitivity Phantom; Data Spectrum Corp.) (8). The innermost tube was of fillable polyethylene with an inside diameter of $1 \mathrm{~mm}$ and outside diameter of $3 \mathrm{~mm}$. The polyethylene tube was filled with an ${ }^{18} \mathrm{~F}$ solution having a specific activity of approximately $2-4 \mathrm{MBq}$ at the start of the PET acquisition and was inserted in all 5 sleeves, which were the same length as each other and had a known amount of attenuation. The tube was positioned in the center of the transaxial FOV, parallel to the axis of the scanner. Five emission scans, each with different attenuation (created by successively removing the outermost sleeves), were performed for $600 \mathrm{~s}$ each. The acquired data were analyzed using the PET Quality Control Tool (PETquact) software (Nihon MediPhysics Co., Ltd.) (9). The acquired true coincidence counting rate was recorded as a function of sleeve thickness and then fitted by linear regression to obtain the extrapolated true counting rate. The system sensitivity was then computed as the ratio between the true counting rate with no attenuation and the activity at the time the scan began. The same measurement was repeated at a $10-\mathrm{cm}$ radial offset from the center of the transaxial FOV.

\section{Evaluation of Image Quality}

A NEMA International Electrotechnical Commission body phantom with 6 spheres of 37-, 28-, 22-, 17-, 13-, and 10-mm inner diameter (Data Spectrum Corp.) was used for this study. The spheres and background can be filled with ${ }^{18} \mathrm{~F}-\mathrm{FDG}$ solution having a 4:1 activity ratio. The background activity was 5.31 and $2.65 \mathrm{kBq} / \mathrm{mL}$. The background activity levels of 5.31 and $2.65 \mathrm{kBq} / \mathrm{mL}$ simulated the normal soft-tissue uptake of patients who received high (7.4 MBq/kg) and low (3.7 MBq/kg) injected doses, respectively (10). PET data were acquired for $10 \mathrm{~min}$ in 3-dimensional listmode and then were reconstructed using ordered-subsets expectation maximization (OSEM) and OSEM plus point-spread function (PSF) plus TOF algorithms. PET images with acquisition times ranging from 1 to $10 \mathrm{~min}$ were then reconstructed. The reconstruction parameters were as follows: 2 iterations, 21 subsets (OSEM + PSF + TOF) or 24 subsets (OSEM), a gaussian filter of $4 \mathrm{~mm}$ in full width at half maximum, and a matrix size of 256 .

To evaluate the PET raw-data quality metric, the noise-equivalent counting rate (NECR) was calculated as follows:

$$
\mathrm{NECR}=(1-\mathrm{SF})^{2} \times(\mathrm{T}+\mathrm{S})^{2} /(\mathrm{T}+\mathrm{S}+[\mathrm{k}+1] \mathrm{R})(\mathrm{kcps}),
$$

where $\mathrm{T}$ is the true coincidence counting rate, $\mathrm{S}$ is the scatter coincidence counting rate, $\mathrm{R}$ is the random coincidence counting rate, $\mathrm{SF}$ is the scatter fraction, and $\mathrm{k}=1$ (noise random correction factor). The true, scatter, and random coincidence counts were obtained from the full sinogram dataset of the PET data acquired over $10 \mathrm{~min}$.

The PET image quality was physically assessed using the coefficient of variance on the background $\left(\mathrm{CV}_{\mathrm{BG}}\right)$. We placed 12 circular regions of interest (ROIs), each $30 \mathrm{~mm}$ in diameter, on the axial slice of the sphere center and on slices $\pm 1 \mathrm{~cm}$ and $\pm 2 \mathrm{~cm}$ away for the background (total, 60 ROIs). The $\mathrm{CV}_{\mathrm{BG}}$ was calculated as follows:

$$
\mathrm{CV}_{\mathrm{BG}}=\left(\mathrm{SD}_{\mathrm{BG}} / \mathrm{C}_{\mathrm{BG}}\right) \times 100(\%),
$$

where $\mathrm{C}_{\mathrm{BG}}$ is the mean activity of the 60 background ROIs and $\mathrm{SD}_{\mathrm{BG}}$ is the mean of the SD of the 60 background ROI counts.

Additionally, the relative recovery coefficients (RC) were evaluated to estimate the image resolution for the 10-min acquisition data (10). All 6 spheres were encompassed by large, circular ROIs on the axial PET images. The relative RC for a j-mm hot sphere $\left(\mathrm{RC}_{\mathrm{j}}\right)$ was calculated as follows:

$$
\mathrm{RC}_{\mathrm{j}}=\mathrm{C}_{\mathrm{j}} / \mathrm{C}_{37}
$$

where $C_{j}$ and $C_{37}$ were the maximum values for a j-mm- and 37-mmdiameter sphere.

TABLE 1

Sensitivities of 39- and 52-Ring PET/CT Scanners

\begin{tabular}{cccc}
\hline Parameter & $\mathrm{D}(\mathrm{cm})$ & $\begin{array}{l}\text { 39-ring } \\
\text { system }\end{array}$ & $\begin{array}{c}\text { 52-ring } \\
\text { system }\end{array}$ \\
\hline Sensitivity (kcps/MBq) & 0 & 5.4 & 9.2 \\
& 10 & 5.9 & 9.4 \\
Total sensitivity & & 5.6 & 9.3 \\
(kcps/MBq) & & &
\end{tabular}

$\mathrm{D}=$ radial distance in from center of FOV . 
TABLE 2

NECR in Relation to Activity Level and Number of Detectors

\begin{tabular}{cc}
\hline Parameter & NECR $(\mathrm{kcps})$ \\
\hline 52-ring high activity & 115.9 \\
52-ring low activity & 59.0 \\
39-ring high activity & 72.0 \\
39-ring low activity & 37.8 \\
\hline
\end{tabular}

\section{Statistical Analysis}

The $\mathrm{CV}_{\mathrm{BG}}$ values were compared by the $t$ test. In all analyses, a $P$ value of less than 0.05 was considered to be statistically significant.

\section{RESULTS}

The system sensitivities of both the 39- and the 52-ring scanners are reported in Table 1 . The system sensitivity of the 52-ring scanner was $66 \%$ higher than that of the 39-ring scanner.

The NECR values are shown in Table 2. The 52-ring scanner increased the NECR by approximately $60 \%$, compared with that observed with the 39-ring scanner. The NECR increased in proportion to the radioactivity levels.

The PET images on both systems at various acquisition times are shown in Figure 2. In terms of background uniformity, the images acquired by the 52-ring scanner were superior to those acquired by the 39-ring scanner. The $\mathrm{CV}_{\mathrm{BG}}$ in relation to acquisition times is shown in Figure 3. The $\mathrm{CV}_{\mathrm{BG}}$ of the 52-ring scanner was superior to that of the 39-ring scanner by approximately $10 \%$ on both activity levels. The $\mathrm{CV}$ of the 52-ring scanner with 3-min acquisition was equivalent to that of the 39-ring scanner with 4-min acquisition $(P=0.08)$.

The relative RC curves for PET images taken using a 10min acquisition are shown in Figure 4. The RC curves for both systems were nearly identical. The effects of the PSF

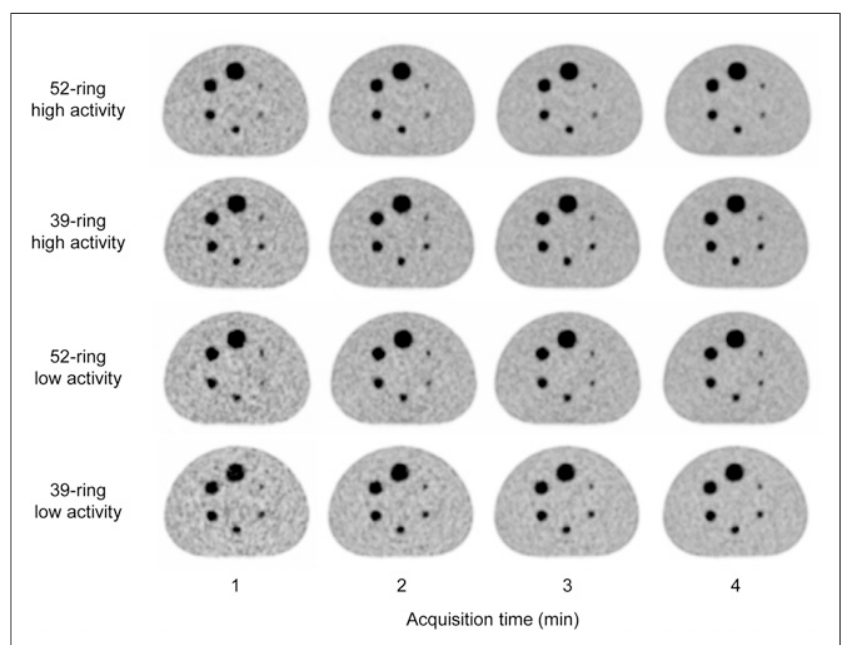

FIGURE 2. PET images reconstructed using OSEM + PSF + TOF with various acquisition times, different activity levels, and 2 types of detector ring. In terms of background uniformity, images acquired using 52-ring scanner were superior to those acquired using 39-ring scanner.

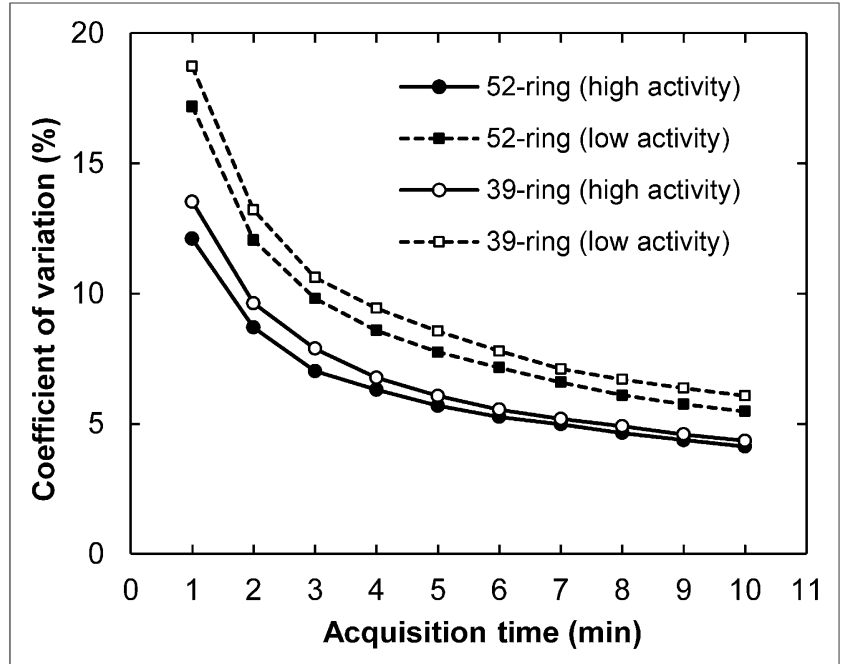

FIGURE 3. CV for OSEM + PSF + TOF images in relation to acquisition times. CV of 52-ring scanner was superior to that of 39 -ring scanner by approximately $10 \%$. CV of 52-ring scanner using 3-min acquisition was equivalent to that of 39-ring scanner using 4-min acquisition.

and TOF for image resolution were also identical, irrespective of the number of detector rings.

\section{DISCUSSION}

The system sensitivity of the 52-ring scanner was $66 \%$ higher than that of the 39-ring scanner. As a result, a higher NECR and better image quality were obtained by the 52ring scanner. In a previous study by Jakoby et al. (5), the NECR of the 52-ring scanner was increased by approximately $50 \%-70 \%$ compared with that of the 39-ring scanner. Our results correspond to those of previous studies. The $\mathrm{CV}_{\mathrm{BG}}$ value of the 52-ring scanner was approximately $10 \%$ lower than that of the 39-ring scanner at both activity levels. The CV of the 52-ring system with a 3-min acquisition time was equivalent to that of the 39-ring system with a 4-min acquisition time. On the basis of these results, the acquisition time per bed position can be reduced by approximately $25 \%$ to obtain the same image quality as the 39-ring scanner. Furthermore, the injected dose can be reduced without compromising image quality.

The RC curves obtained by the 2 scanners were almost identical. Jakoby et al. also reported that the spatial resolution was the same for both 39- and 52-ring scanners without TOF information $(5,7)$. The correspondence of the RC was expected to be attributable to the use of identical geometry detectors. Thus, in clinical practice, the standardized uptake value is considered to be independent of the number of detector rings. For both the 39- and the 52-ring scanners, the PSF and TOF improved the RC of the PET images as well. Our results indicated that the effects of PSF and TOF information on the standardized uptake value were independent of the number of detector rings (11).

The axial FOV of the 52-ring scanner was $5.4 \mathrm{~cm} \mathrm{(33 \% ),}$ longer than that of the 39-ring scanner. A large axial FOV 


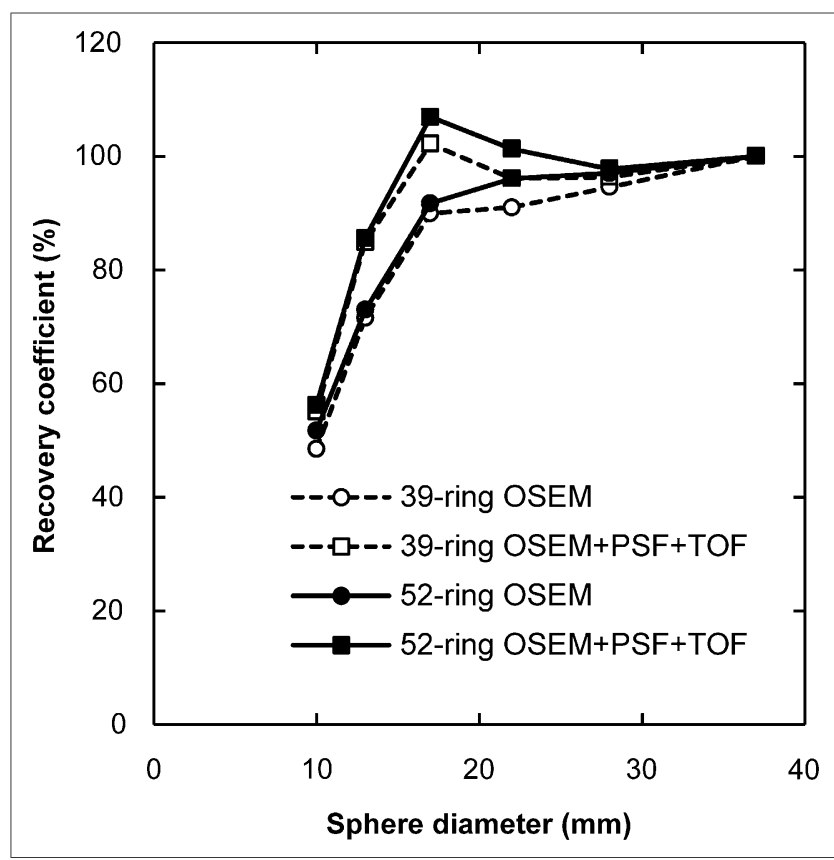

FIGURE 4. Relative RC curves at 10-min acquisition in relation to reconstruction methods and number of detector rings. Although PSF and TOF also increased RC, RC curves were nearly identical independent of number of detector rings.

can scan over a wide range at one time. Therefore, the number of bed positions required to scan from the mid thigh to the top of a patient using the 52-ring scanner was approximately $70 \%$ of that using the 39-ring scanner. Furthermore, the increase in sensitivity allowed for a $25 \%$ decrease in the acquisition time without compromising image quality. Thus, the total acquisition time for a multibed acquisition can be shortened to $53 \%(0.7 \times 0.75=0.53)$ for the 52 -ring scanner.

In this study, we evaluated the sensitivity, NECR, and CV of the phantom uniform area. The sensitivity evaluation was performed only among the NEMA NU-2 standard because we expect that the sensitivity was dependent on the number of detector rings. Further examinations to evaluate all units of the NEMA NU-2 standard are recommended.

\section{CONCLUSION}

The image quality of the 52-ring scanner is superior to that of the 39-ring scanner. Furthermore, the whole-body scanning time can be reduced by approximately $40 \%$ without compromising image quality. The spatial resolution was the same for both scanners. Thus, the obtained standardized uptake value is constant irrespective of the number of detector rings.

\section{DISCLOSURE}

No potential conflict of interest relevant to this article was reported.

\section{ACKNOWLEDGMENTS}

We thank the staff of the Department of Clinical Radiology and Medical Technology at Kyushu University Hospital and Saga University Hospital for their valuable clinical support.

\section{REFERENCES}

1. Rohren EM, Turkington TG, Coleman RE. Clinical applications of PET in oncology. Radiology. 2004;231:305-332.

2. Gupta N, Price PM, Aboagye EO. PET for in vivo pharmacokinetic and pharmacodynamics measurements. Eur J Cancer. 2002;38:2094-2107.

3. Halpern BS, Dahlbom M, Quon A, et al. Impact of patient weight and emission scan duration on PET/CT image quality and lesion detectability. J Nucl Med. 2004;45:797-801.

4. MacDonald LR, Harrison RL, Alessio AM, Hunter WC, Lewellen TK, Kinahan PE. Effective count rates for PET scanners with reduced and extended axial field of view. Phys Med Biol. 2011;56:3629-3643.

5. Jakoby BW, Bercier Y, Watson CC, et al. Performance characteristics of a new LSO PET/CT scanner with extended axial field-of-view and PSF reconstruction. IEEE Trans Nucl Sci. 2009;56:633-639.

6. Jonsson C, Odh R, Schnell P-O, Larsson SA. A comparison of the imaging properties of a 3- and 4-ring Biograph PET scanner using a novel extended NEMA phantom. In: Nuclear Science Symposium Conference Record, 2007. Piscataway, NJ: IEEE; 2007;4:2865-2867.

7. Jakoby BW, Bercier Y, Conti M, Casey ME, Bendriem B, Townsend DW. Physical and clinical performance of the mCT time-of-flight PET/CT scanner. Phys Med Biol. 2011;56:2375-2389.

8. NEMA Standards Publication NU 2-2007: Performance Measurement of Positron Emission Tomographs. Rosslyn, VA: National Electrical Manufacturers Association; 2007.

9. Matsumoto K, Endo K. Development of analysis software package for the two kinds of Japanese fluoro-D-glucose-positron emission tomography guideline [in Japanese]. Nihon Hoshasen Gijutsu Gakkai Zasshi. 2013;69:648-654.

10. Fukukita H, Senda M, Terauchi T, et al. Japanese guideline for the oncology FDG-PET/CT data acquisition protocol: synopsis of version 1.0. Ann Nucl Med. 2010;24:325-334.

11. Akamatsu G, Mitsumoto K, Taniguchi T, Tsutsui Y, Baba S, Sasaki M. Influences of point-spread function and time-of-flight reconstructions on standardized uptake value of lymph node metastases in FDG-PET. Eur J Radiol. 2014;83: 226-230. 